combination antibiotics for multidrug-resistant (MDR)-Pseudomonas aeruginosa infections are limited. Methods: This retrospective, case-control observational study was based on chart review of the patients managed at the University of Kentucky. Results: In total, 143 patients with MDRO Pseudomonas aeruginosa infections were identified and divided into 2 groups: 1 group received newer BLBI combinations with or without aminoglycosides or polymyxins, for at least 72 hours, and the control group received carbapenem containing combination antibiotics or other antibiotics. Baseline characteristics and patient outcomes are shown in Table 1. Discussion: The newer BLBI combinations group consisted of $60.8 \%$ MDR Pseudomonas bacteremia, whereas the control group had $68.4 \%$ of MDR Pseudomonas respiratory cultures. Overall, the use of newer BLBI combinations such as ceftazidime/avibactam, ceftolozane/tazobactam, and meropenem/vaborbactam was associated with lower rates of acute kidney injury (AKI), shorter LOS, and lower mortality rates compared to the control group, and these differences were statistically significant. Because the 2 populations of patient differed significantly based on the site of infection (sepsis vs pneumonia), the data were reanalyzed to evaluate the impact of therapy on the occurrence of AKI, LOS, and mortality based on the site of infection. Only those patients with sepsis who received the newer combination drugs had significantly better rates of AKI, lower LOS, and had lower rates of mortality. The 2 treatment arms were not statistically different when comparing patients with pneumonia. Additionally, the use of these new combination therapies did not make a difference regarding readmission rates or duration of bacteremia for the patients included in the study.

Funding: No

\section{Disclosures: None}

Antimicrobial Stewardship \& Healthcare Epidemiology 2021;1(Suppl. S1):s69-s70

doi:10.1017/ash.2021.134

\section{Presentation Type:}

Poster Presentation

Subject Category: MDR GNR

Time Above All Else: Pharmacodynamic Analysis of B-Lactams in Critically Ill Patients

Katherine Landmesser; David Burgess and Justin Clark

Background: Despite the development of new $\beta$-lactam agents, gram-negative resistance continues to be an increasing concern in the healthcare setting. The understanding and optimizing antimicrobial pharmacokinetics and pharmacodynamics are essential to enhance activity of appropriate therapy, improve clinical outcomes, and reduce the development of resistance. Methods: A pharmacodynamic analysis was performed for $4 \beta$-lactams (aztreonam, cefepime, piperacillin/tazobactam, and meropenem) and 14 dosage regimens as either intermittent bolus (IB) or prolonged infusion (PI) against 7 gram-negative pathogens: Klebsiella pneumoniae, K. oxytoca, Escherichia coli, Enterobacter cloacae, E. aerogenes, Acinetobacter baumannii, and Pseudomonas aeruginosa. Unit-specific minimum inhibitory concentration (MIC) distribution data were generated using antibiogram data over a decade for 4 intensive care units within our institution: medical ICU, cardiovascular ICU, surgical ICU, and neurosurgical ICU. Published pharmacokinetic parameter estimates in critically ill patients, combined with this MIC distribution data, were utilized to perform Monte Carlo simulations for each antimicrobial regimen. The percentage of time for which the unbound concentration of antibiotic remained above the MIC ( $f$ T $>$ MIC) was utilized as the pharmacodynamic target for each agent: $40 \% f \mathrm{~T}>\mathrm{MIC}$ for meropenem, $50 \% f \mathrm{~T}>\mathrm{MIC}$ for piperacillin/tazobactam, $60 \% f \mathrm{~T}>\mathrm{MIC}$ for aztreonam, and $70 \% f \mathrm{~T}>\mathrm{MIC}$ for cefepime. Regimens were modeled using Oracle Crystal Ball software to determine the likelihood of achieving $>90 \%$ probability of target attainment (PTA). Because resistance rates were significantly higher for $P$. aeruginosa and A. baumannii, cumulative PTAs for K. pneumoniae, K. oxytoca, E. coli, E. cloacae, and E. aerogenes were analyzed separately to determine the relative PTA for Enterobacterales in each ICU. Results: No intermittent infusion regimens of piperacillin/tazobactam, aztreonam, or cefepime achieved $>90 \%$ PTA for any organism. Piperacillin/ tazobactam $4.5 \mathrm{~g}$ infused over 4 hours (PI q6h) and aztreonam $2 \mathrm{~g}$ PI q6h failed to achieve adequate PTA for Enterobacterales with only $84 \%$ and $85 \%$ PTA, respectively. For Enterobacterales, the only regimens to achieve $>90 \%$ PTA included cefepime $2 \mathrm{~g}$ infused over 3 hours (PI q8h) and meropenem 1g IB q8h with $95 \%$ and $99 \%$ PTA, respectively. Meropenem $2 \mathrm{~g}$ PI q $8 \mathrm{~h}$ was the only regimen capable of achieving $>90 \%$ PTA for both A. baumannii and P. aeruginosa with $97 \%$ and $92 \%$ PTA, respectively. Conclusions: Although utilization of high doses and prolonged infusions dramatically improve the pharmacodynamics of $\beta$-lactam therapy, the only regimen capable of achieving adequate PTA for all organisms analyzed was meropenem $2 \mathrm{~g}$ PI q8h. To reduce carbapenem use, combination therapy may be considered for critically ill patients receiving aztreonam, cefepime, or piperacillin/tazobactam for empiric treatment of gram-negative infections.

Funding: No

Disclosures: None

Antimicrobial Stewardship \& Healthcare Epidemiology 2021;1(Suppl. S1):s70 doi:10.1017/ash.2021.135

\section{Presentation Type:}

Poster Presentation

Subject Category: MDR GNR

Difference in Susceptibility Between Carbapenemase- and NonCarbapenemase-Producing Carbapenem-Resistant Enterobacteriaceae Justin Clark and David Burgess

Background: Carbapenem-resistant Enterobacteriaceae (CRE) remain among the most urgent infectious threats according to the CDC Threats Reports. Although focus has often been placed on carbapenemase-producing phenotypes, there is increasing interest in distinguishing the optimal treatment and outcomes of carbapenemase-producing (CP) and non-carbapenemaseproducing (NCP) CRE. We compare antimicrobial susceptibility patterns between CP-CRE and NCP-CRE isolated from patients at our academic medical center. Methods: All CRE isolates of Enterobacter cloacae, Escherichia coli, Klebsiella aerogenes, $K$. oxytoca, and $K$. pneumoniae in adult inpatients from 2010 to 2019 were included in this study. Susceptibility testing was performed using the BD Phoenix Automated System (BD Diagnostics, Sparks, MD). CLSI susceptibility break points were utilized in the susceptibility analyses of all antimicrobials tested. To determine carbapenemase production, isolates resistant only to ertapenem were considered NCP-CRE, and those resistant to both ertapenem and meropenem were considered CP-CRE. Statistical comparisons of susceptibility profiles were performed using either the $\chi^{2}$ test or the Fisher exact test. All data preprocessing and statistical analyses were performed using Python software. Results: Over the decade, we identified 291 CRE isolates (216 isolates resistant only to ertapenem and 75 resistant to ertapenem and meropenem). The ertapenem-resistant-only phenotype comprises $\sim 66 \%$ of the total CRE population and is largely composed of E. cloacae (67\%). As expected, most $\beta$-lactam susceptibilities were negligibly low between the 2 groups; however, other clinically relevant antimicrobials (aminoglycosides, fluoroquinolones, and sulfamethoxazole/trimethoprim) exhibited starkly different susceptibility profiles ( $P$ value Conclusions: These findings suggest that the most predominant CRE phenotype at our institution is not carbapenemase production. Evaluation of outcomes between CP- and NCP-CRE should be pursued further. The large differences in the MIC distributions may lead to differing outcomes for the affected patients.

Funding: No

Disclosures: None

Antimicrobial Stewardship \& Healthcare Epidemiology 2021;1(Suppl. S1):s70

doi:10.1017/ash.2021.136

Presentation Type:

Poster Presentation

Subject Category: MDR GNR

Stewardship Nightmare: Ceftolozane/Tazobactam-Resistant Pseudomonas aeruginosa Infections Accelerated by the COVID-19 Pandemic

Adam Haviland; Gregory Weston; Priya Nori; Wendy Szymczak; Yi Guo and Rebecca M. Marrero Rolon

Background: The Centers for Disease Control and Prevention reported 32,600 cases, 2,700 deaths, and healthcare costs of 767 million dollars (c) The Author(s), 2021. Published by Cambridge University Press on behalf of The Society for Healthcare Epidemiology of America. This is an Open Access article, distributed under the terms of the Creative Commons Attribution licence (http://creativecommons.org/licenses/by/4.0/), which permits unrestricted re-use, distribution, and reproduction in any medium, provided the original work is properly cited.

S70 2021;1 Suppl 1 